\title{
The Glitter of Carbon Nanostructures in Hybrid/Composite Hydrogels for Medicinal Use
}

\author{
Daniel Iglesias, Susanna Bosi, Michele Melchionna, Tatiana Da Ros and Silvia Marchesan*
}

Department of Chemical and Pharmaceutical Sciences, University of Trieste, Trieste, Italy

\begin{abstract}
In recent years, we have witnessed to fast developments in the medicinal field of hydrogels containing various forms of integrated nanostructured carbon that adds interesting mechanical, thermal, and electronic properties. Besides key advances in tissue engineering (especially for conductive tissue, such as for the brain and the heart), there has been innovation also in the area of drug delivery on-demand, with engineered hydrogels capable of repeated response to light, thermal, or electric stimuli. This mini-review focusses on the most promising developments as applied to the gelation of protein/peptide (including self-assembling amino acids and low-molecular-weight gelators), polysaccharide, and/or synthetic polymer components in medicine. The emerging field of graphene-only hydro-

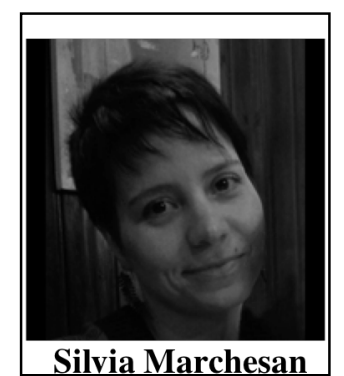
gels is also briefly discussed, to give the reader a full flavor of the rising new paradigms in medicine that are made possible through the integration of nanostructured carbon (e.g., carbon nanotubes, nanohorns, nanodiamonds, fullerene, etc.). Nanocarbons are offering great opportunities to bring on a revolution in therapy that the modern medicinal chemist needs to master, to realise their full potential into powerful therapeutic solutions for the patient.
\end{abstract}

Keywords: Carbon nanostructures, carbon nanotubes, composite, graphene, hybrid, hydrogels.

\section{INTRODUCTION}

Hydrogels are soft materials that retain a high level of water to find application in the medicinal field especially as absorption or release media, e.g., for tissue engineering, wound healing, drug delivery [1]. They can be made of a chemical or physical network of molecules, be it protein/peptides [2], polysaccharides [3], or synthetic polymers [4]. Incorporation of nanomaterials is a useful means to achieve unprecedented performance by adding new, interesting features [5]. In particular, carbon nanostructures (e.g., single- or multi-wall carbon nanotubes or SWCNT/ MWCNT, graphene, carbon nanohorns, nanodiamonds, fullerenes, Fig. 1) add very interesting mechanical and electronic properties, thanks to their unique chemical structure [6]. However, there is always a certain level of concern for biological use of carbon nanostructures, as especially CNTs. First of all, the risk for their aggregation into asbestos-like pathogenic aggregates, although it is now known this is completely alleviated upon appropriate chemical functionalization [7]. Other reasons for concern are: the risk for immunogenic response, oxidative stress, and prolonged accumulation in living tissue, thus eliciting inflammation or even more serious adverse reactions [8].

Nevertheless, there is a growing body of work showcasing nanocarbon innovative potential in nanomedicine, especially upon appropriate chemical functionalization [9]. Besides, in vitro studies on CNT-containing hydrogels evidence no sign of cytotoxicity on intestinal cells, showing promise for this class of materials [10]. CNT addition to hydrogels

*Address correspondence to this author at the Department of Chemical and Pharmaceutical Sciences, University of Trieste, Via Giorgieri 1, 34127 Trieste, Italy; E-mail: smarchesan@units.it opens the doors to "smart" biomaterials capable to respond to diverse stimuli, e.g., for drug release [11]. We will see that also graphene $(\mathrm{G})$ is being increasingly studied to achieve similar properties, while other carbon nanoforms have only received limited interest in nanomedicine, thus far.

This mini-review focusses on the latest exciting developments on this class of biomaterials, be it hybrid or composites, for applications in the biomedical field. Due to the vast amount of literature on the topic, we will neither review the field of sensors, electrodes, actuators, nor will we attempt to give a comprehensive survey of the topic.

By contrast, only the most promising and innovative studies for medicinal use of the last few years will be discussed, in an attempt to delineate the future directions of the field, and, especially, the high potential that can be realised in therapy thanks to the incorporation of carbon nanostructures. For instance, thanks to their high aspect ratio, CNTs offer the possibility to transport high levels of cargo (e.g., imaging agents, or drugs), as well as to be functionalized with antibodies to cap receptors for cell targeting (e.g., cancer cells) with subsequent CNT internalization and/or cell death, and, finally, to be rapidly eliminated via renal excretion [12]. Therefore, it is apparent that carbon nanostructures open new doors to innovative therapy paradigms that were simply unthinkable a decade ago and that are moving at a fast pace [13].

\section{PROTEIN AND PEPTIDE HYDROGELS}

\subsection{Protein Hydrogels}

Protein hydrogels are a plausible choice for biomaterials, thanks to their biocompatibility and biodegradability. They typically exploit the self-assembling ability of extracellular 


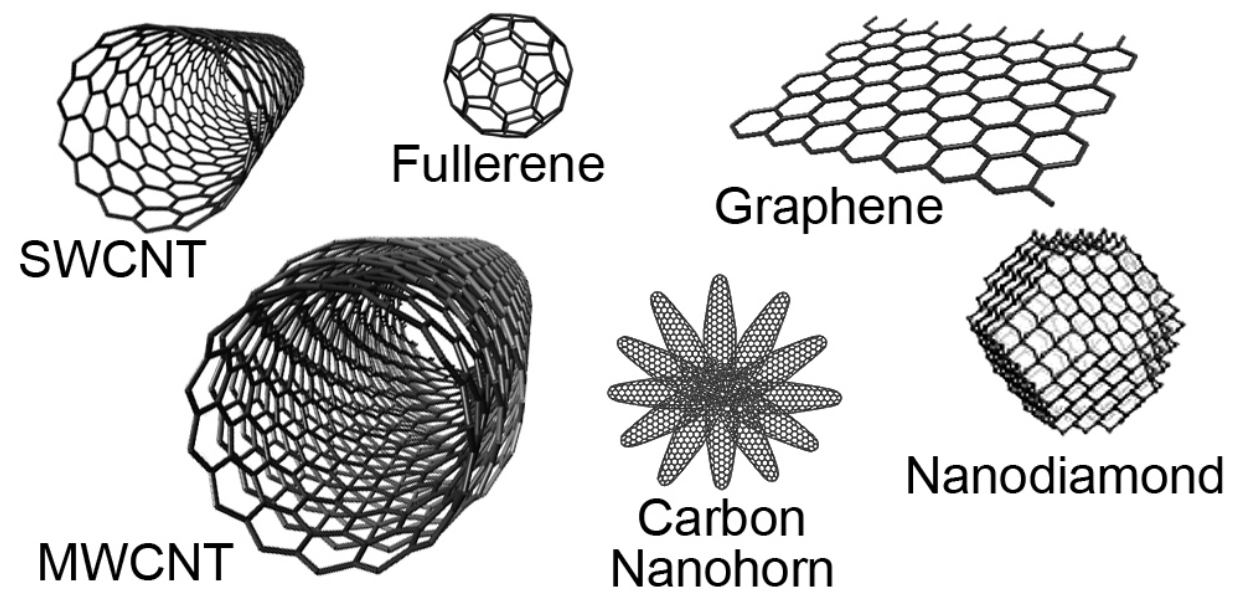

Fig. (1). Various forms of nanostructured carbon (for clarity, not to scale).

matrix (ECM) components, such as collagens and gelatin [2]. However, they are often obtained from an animal source and display well-known issues of purification, immunogenicity, or even risk of pathogen transfer. Therefore, even though this is a mature field of research, protein hydrogels have been rarely admitted to clinical trials [2].

Nevertheless, viscoelastic and biological properties of protein hydrogels make them stand out for medicinal use, and they are often used as components of more sophisticated biomaterials. In addition, amphiphilic proteins are wellknown to be good dispersants of hydrophobic nanocarbons, and their mutual interactions can be exploited to achieve advanced biomaterials [14]. Their combination with carbon nanostructures is a useful means to leverage their mechanical weakness, while adding conductive properties, thus extending their potential use to match the viscoelastic (and electronic properties) of different human tissues. SWCNTs can be used as a fibrous additive to $3 \mathrm{D}$ cell-seeded protein hydrogels to increase the electrical conductivity of such composites [15].

For example, $\beta$-lactoglobulin is a gelling protein that it is widely used in the food industry [2]. It can form hybrids with MWCNTs to gel at protein concentrations that are notably lower than those required by the protein alone. Hybrids of similar nanomorphology are observed when the protein is bound via electrostatic interactions to either covalently, or non-covalently, sulfonated CNTs (Fig. 2) [16], suggesting a fibrillization promoting role played by the nanotubes, as seen in the case of the interaction between CNTs and amyloids [17]. Reversible gelation is possible thanks to the protein component, which gels at $\mathrm{pH}<\mathrm{pI}$ (i.e., the protein isoelectric point) and flows at $\mathrm{pH}>\mathrm{pI}$, and the presence of CNTs increases the gel elastic modulus [16].

Silk fibroin is another gelling protein that has the advantage of having a hydrophobic character to favourably interact with carbon nanostructures. Besides, thanks to its amphiphilic nature, the protein can establish hydrogen bonds with GO. As a result, silk fibroin forms composites with graphene oxide (GO) to yield hydrogels of increased tensile strength, modulus, and failure strain, which thus display overall improved mechanical properties for their use as tissue engineering scaffolds [18].
In the case of engineered cardiac tissue, gelatin-SWCNT composite hydrogels can be prepared by mixing of the two components, followed by glutaraldehyde crosslinking. These biomaterials can integrate with host myocardium after implant, and, remarkably, inhibit further pathological deterioration in an infarct model. Cells preferably adjoin SWCNTs throughout the infarct area, and show increased spontaneous activity (relative to the gelatin control) taking advantage of the CNT conductive properties [19].

In addition to tissue engineering scaffolds, also endovascular stent devices can take advantage of CNTs. For instance, a nanobiohybrid hydrogel-based vascular stent device can be engineered to promote endothelial recovery in a sitespecific manner, thanks to combined effects. A balloonexpandable stent metal structure can be embedded in a fibrin hydrogel that is assembled layer-by-layer with intercalation of CNTs and nanoparticles carrying DNA (encoding for specific growth factors). In such a sophisticated design, the CNTs fine-tune the gel mechanical properties, while reducing loss of therapeutics away from the site of action, thanks to electrostatic interaction between the negatively-charged, polyacrylic acid-wrapped CNTs and the positively-charged, gene-carrying nanoparticles. Importantly, no inflammation is observed following in vivo stent application [20].

The combination of protein and carbon nanostructures into a hydrogel also allows to fine-tune the material properties by carefully balancing the amount of each component. For instance, hydrogels formed by soybean protein and GO present a porosity that is increased by the protein thanks to its hydrophilic character, thus ability to retain water [21]. These materials are interesting candidates for drug delivery, as shown by using the antibiotic ciprofloxacin as a model [21].

It is interesting to note that in some cases the interaction between carbon nanostructures and biological gel matrices can be detrimental for the mechanical properties of the latter, however, this behavior can be exploited for biomedical purposes. In fact, it has been demonstrated that fullerene derivatives, metallofullerol $\mathrm{Gd} @ \mathrm{C}_{82}(\mathrm{OH})_{22}$, and the $\mathrm{C}_{60}(\mathrm{OH})_{24}$ analogue, are able to decrease the stiffness of collagen. The so-induced fragility of the biological system leads to decreased cell adhesion and viability, allowing envisaging a 

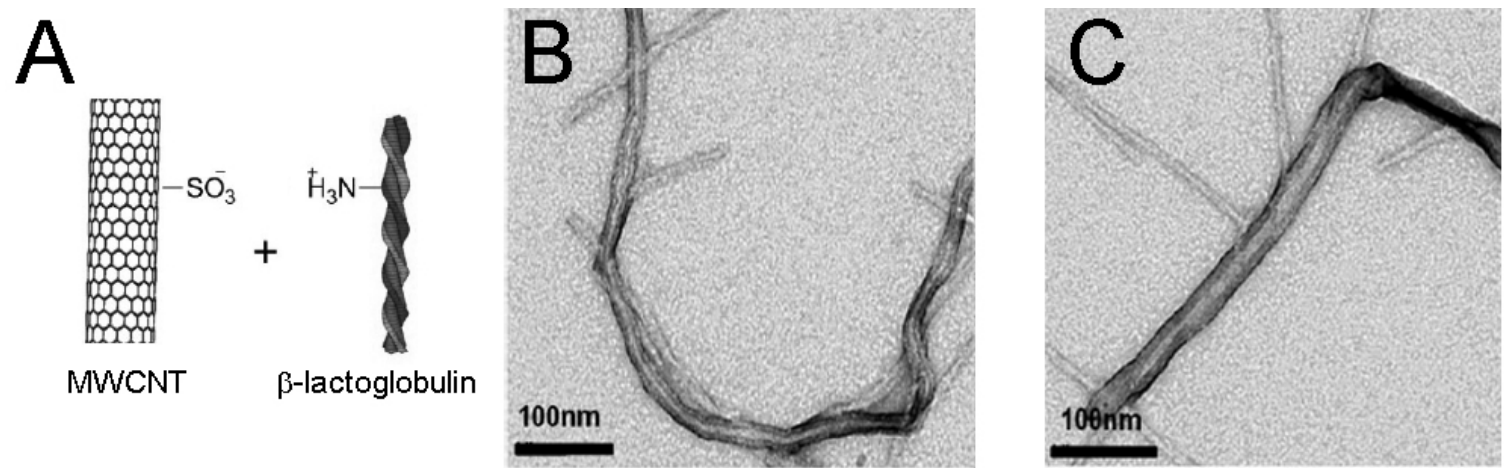

Fig. (2). A) Schematic representation of the electrostatic interaction between functionalized MWCNTs and $\beta$-lactoglobulin. B-C) TEM images of hybrids formed with the protein and either covalently (B) or non-covalently (C) sulfonated MWCNTs. Adapted with permission from Ref. [16], Copyright (C) 2012, American Chemical Society.

new use of these nanomaterials in the treatment of tumor tissues where the decreased rigidity of the extracellular matrix, composted also by collagen, could end up with a slower metastasis process [22].

\subsection{Polypeptide Hydrogels}

As mentioned in the section above, natural proteins present some disadvantages for applications in the medicinal field, thus there is a certain research effort to substitute them with polypeptides of synthetic origin. Peptides allow for batch-to-batch high reproducibility in terms of chemical composition, and their chemistry is well-established and versatile. In addition, their structure is more resilient relative to complex macromolecular proteins that require correct folding for function, and that are more sensitive to environment conditions [2]. Finally, amphiphilic peptides can effectively disperse and stabilize CNTs in aqueous solutions [14], and also in hydrogels [23], by establishing hydrophobic contacts with the nanostructure and exposing the hydrophilic portions for interaction with water. Therefore, instead of using fulllength gelling proteins, selected peptide fragments are often preferred.

For instance, the silk fibroin-derived Gly-Ala-Gly-AlaGly pentamer can be coupled to a GO-binding pyrene unit and a cross-linkable tyrosine residue (Fig. 3) to achieve an injectable "smart" hydrogel. This hybrid material has excellent mechanical properties and recovers well, after repeated gel-disruptive cycles of applied strain. Thanks to the nearinfrared (NIR) responsive behavior of GO, it is possible to obtain on-demand drug release upon gel irradiation at 808 $\mathrm{nm}$, as the GO absorbs light, leading to a local temperature increase that reversibly unfolds the gel. Repeated cycles of doxorubicin drug release prove therapeutically effective in vivo against a tumor model in nude mice, while not showing signs of cytotoxicity arising from leaching of the gel components [24].

Elastin-like polypeptides (ELPs) are a valuable class of hydrogelling agents owing to their thermoresponsive behavior. At low temperature, ELPs adopt a random coil configuration, while raising the temperature decreases the solubility and proteins aggregate into fibrillar structures. These properties, together with ease of production also by recombinant techniques, makes them popular vehicles for drug delivery and biomaterials [25]. Recombinant ELPs can be genetically encoded to be fused with other peptide motifs, for instance binding graphene, to combine the peptide thermo-behavior with graphene infrared photo-response, thus yielding smart hybrids with dual control [26].

A cyclic, hydrogel-forming decapeptide can been used to solubilize fullerene by means of ultra-high vibration ball milling, thus obtaining a water-soluble nanocomposite based on $\pi-\pi$ interactions. Despite the fact that this complex changes the gelation properties of the peptide, since in the presence of $\mathrm{C}_{60}$ precipitation occurs, the proposed approach results to be very useful to prepare colloidal dispersions for biomedical uses [27].

\subsection{Self-Assembling Ultra-Short Peptide Hydrogels}

Peptides are typically prepared by solid-phase methods, which offer advantages in terms of ease of purification, but at the expense of an elevated cost and solvent waste, which increase exponentially with peptide length. This is why for biomaterials and drug delivery use it is economically more viable to use ultrashort peptides, made of up to 3 amino acids, that can be prepared also by alternative methods [28, 29]. The commercial potential of gelling, ultra-short peptides for medicinal use is evident from patent surveys [30]. Besides, even single amino acids can yield hydrogels, given that they are functionalized with sufficiently hydrophobic moieties, such as fluorene derivatives, allowing for molecular stacking into ordered supramolecular structures. As an example, Fmoc-Phe forms hydrogels at physiological $\mathrm{pH}$ through the formation of $\pi-\pi$ interactions between the aromatic units. Such interactions can be exploited also to incorporate $\mathrm{sp}^{2}$-carbon nanostructures into the soft material. With the aid of ultrasonication, oxidised SWCNTs can be dispersed and aligned with Fmoc-Phe nanofibers, to yield a hybrid hydrogel. The conductive material is more elastic and thermally stable than the gel made by the peptide alone, giving scope for use in medicinal applications [31]. Similarly, Fmoc-Phe-Asp-OH and Fmoc-Tyr-Asp-OH hydrogels are able to establish $\pi-\pi$ interactions with rGO sheets, resulting in a nanohybrid material of enhanced rigidity [32]. A noncovalent mechanochemical approach is also possible to disperse and incorporate carbon nanostructures (including graphene and fullerenes) into a self-assembled short peptide hydrogel [33]. 
A PyGAGAGY peptide<smiles>C[C@H](NC(=O)CNC(=O)CCCc1ccc2ccc3cccc4ccc1c2c34)C(=O)NCC(=O)NCC(=O)N[C@@H](C)C(=O)N[C@@H](Cc1ccc(O)cc1)C(=O)O</smiles>

Graphene binding motif

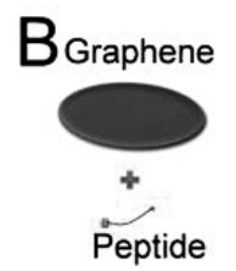

GA repeats

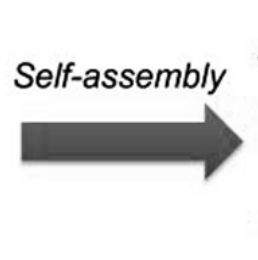

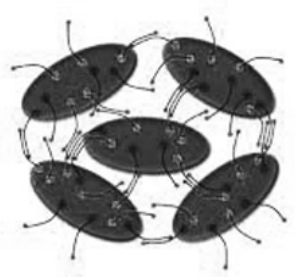

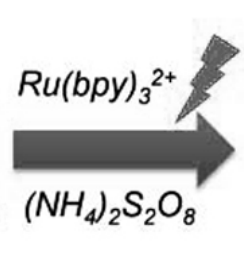

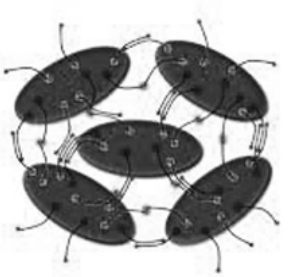

Fig. (3). A) Silk fibroin-derived peptide sequence. B) Schematic representation of the gelling mechanism based on peptide self-assembly and tyrosine photo-crosslinking. Reproduced from Ref. [24] with permission of The Royal Society of Chemistry.

Glutathione (GSH) is a tripeptide that is an interesting building block for biomaterials, considering its physiological role of free radicals scavenger. GO can be used as templating agent for the self-assembly and gelation of glutathione, through the formation of colloidal particles that stack into anisotropic fibers. Depending on conditions applied, different nanomorphologies can be obtained for use in drug delivery, including bead-like structures (Fig. 4) arising from the self-scrolling of GO. Upon thermal treatment, the structures become hollow, although their morphology is preserved [34].

\subsection{Future Directions of Protein/Peptide Hydrogels}

Proteins have historically been elected components to achieve hydrogel biomaterials, however, recent trends prefer peptide substitutes, thanks to their higher batch-to-batch reproducibility and chemical resilience. Therefore, there is a notable research effort devoted towards the identification of peptide motifs with specific protein-mimicking function, and even more so, in the case of self-assembling low-molecularweight components. Since peptide production cost grows exponentially with its amino acid sequence length, a very recent research area focusses on di- and tri-peptides as economically viable solutions for biomaterials [28].
Both proteins and peptides are well-known to favourably interact with nanostructured carbon to help its homogeneous dispersibility in aqueous media [14]. However, thus far, there are only limited studies on their combined use for hydrogel formation, especially for medicinal use. In light of the fast developments towards self-assembling short peptide hydrogels on one hand, and nanostructured carbon functionalization for improved biocompatibility on the other, it is expected that their combined use will be the target of much more attention in near future studies.

\section{CARBOHYDRATE HYDROGELS}

Among the numerous macromolecules that can be used for hydrogels, polysaccharides are extremely advantageous, being widely present in living organisms and often being produced by recombinant DNA techniques. Coming from renewable sources, they typically offer an economical advantage over synthetic polymers. Polysaccharides are generally well-tolerated in vivo and their physico-chemical properties make them suitable for many applications, spanning from tissue regeneration (particularly for cartilage), through drug delivery devices, to gel entrapment systems for the immobilization of cells. In addition, they can be used also for targeting purposes, for instance, polysaccharide-wrapped CNTs can selectively deliver doxorubicin to cancer cells, exploiting
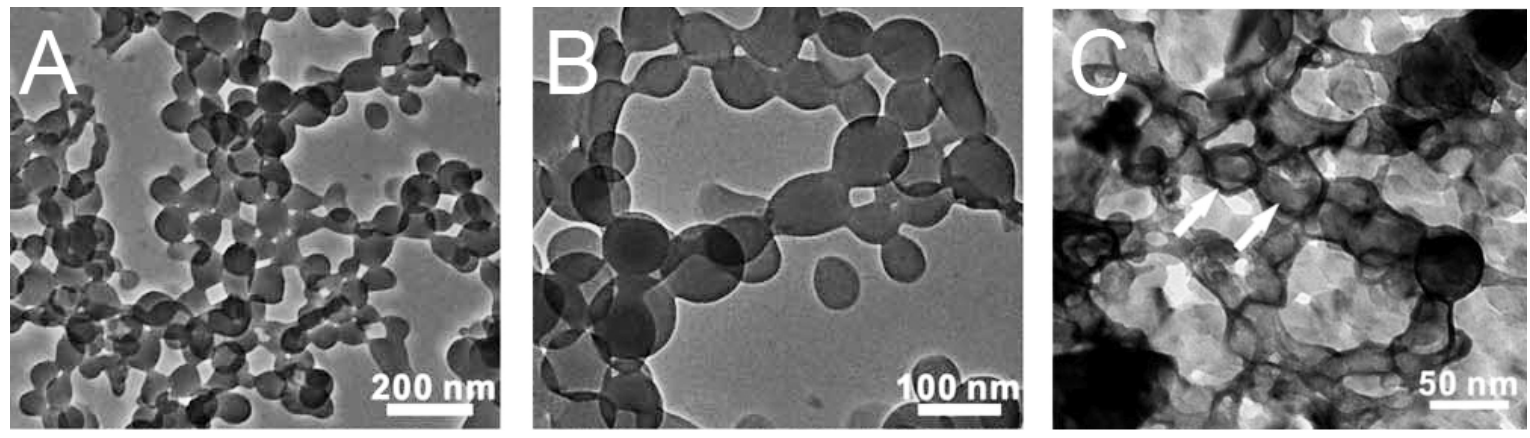

Fig. (4). TEM images of GSH-GO hydrogels reveal a bead-like nanomorphology at both low (A) and high (B) magnification that is preserved after thermal treatment (C). Adapted from ref. [34] with permission from The Royal Society of Chemistry. 
chitosan to assist with CNT dispersibility, and hyaluronan to selectively target CD44 on cancer cells [35].

There are many polysaccharides used to form gel biomaterials. Chitosan, alginate, hyaluronic acid, cellulose, and agarose, are the most popular options (Fig. 5). However, hydrogels with improved mechanical properties are required during tissue regeneration to provide support and mechanical signaling to cells both in vitro and in vivo, for functional optimization of the new extracellular matrix being formed. In this context, functionalized water-soluble carbon nanostructures can be very good candidates to reinforce the biopolymer hydrogels without altering their chemical properties. The interplay of the physical properties of CNTs with a biopolymer can give birth to a new generation of implants to reconstruct natural tissues that are subjected to high mechanical stress, or that require electrical and thermal conductivity at physiological state [36].

Indeed, CNTs incorporated with biopolymers have shown great biocompatibility for orthopedic implants [37]. Graphene can also contribute to confer good electric and thermal conductivity, as well as mechanical stiffness [38]. At present, literature on graphene polymer nanocomposites for biomedical applications is still very scarce.

Besides the use of the materials containing carbon nanostructures, one has to consider their fate after expletion of the desired function. In general, polysaccharide-based hydrogels are mostly biodegradable and bioresorbable. On one hand, this is an advantage as in the case of drug delivery devices. On the other, it can become a drawback if the toxicity of the carbon nanomaterial itself is not well established or it is suboptimal. Nevertheless, in line of principle both the presence of the carbon nanomaterial, and the modified chemical nature of the polymer to interact with it, will affect the degradation profile of the hydrogel, and will likely increase the stability of the whole structure [39].

\subsection{Chitosan Hydrogels}

Chitosan (CS) is one of the most common polysaccharides that has been conjugated with carbon nanomaterials. One common approach is the covalent binding of CS amine groups with activated carboxylic functions of oxidized CNTs [40]. CS is a natural polysaccharide derived from chitin with a wide range of both current and potential applications in biology, medicine, and biotechnology [41]. In literature, there is a number of studies where CNTs have been integrated into a CS matrix for several purposes. In general, the incorporation of CNTs into chitosan results in an improvement of mechanical [42] and thermal [40] properties. Typically, these composites are aimed for tissue engineering, especially for bone [43], and cartilage tissue replacement, but also for nerve tissue reparation [44]. One of the major problems in the preparation of CS with CNT materials is the difficulty to disperse the CNTs in the polymer matrix. Several methods have been used for this purpose, such as controlled surface deposition and cross-linking [45], solution evaporation [46], thermally-induced phase separation followed by freeze-drying [47], electrodeposition [48], layerby-layer spraying [49], $\mathrm{pH}$ and electrical actuation [50], and wet spinning [51].

MWCNT-CS nanoparticle hybrids can be synthesized in situ through ionotropic gelation, which is an extremely mild process that involves the mixing of two aqueous solutions at room temperature. Under optimal conditions, CS nanoparticles can be tethered to the MWCNT surface in high density and with relatively uniform coverage, yielding hybrids of good dispersibility and stability in aqueous solutions. The materials possess a higher BSA immobilization efficiency (77\%), than MWCNTs alone (43\%). It is expected that these hybrids may be used as biocompatible protein carriers, with potential applications as biomolecule delivery systems in gene/protein therapy [52]. Alternatively, gelatin can be used to facilitate CNT dispersion and achieve a homogeneous gelatin-chitosan-CNT hydrogel. The conductive material has great potential in cardiac tissue engineering; presence of SWCNTs increases cell electrical excitability and leads to the generation of more mature action potentials. In contrast with scaffolds suffering from poorly adjuncted cardiomyocytes that generate arrhythmias, in this case cells infiltrate well the scaffold to nearly attain the natural heartbeat frequency, giving hope for future use of the material to achieve myocardial patches [53].

An interconnected network structure of organicinorganic scaffold can be attained also by other means, such as through the covalent linkage of the carboxyl group of functionalized carbon nanohorns, with the amine group of CS. The good dispersibility of functionalized nanostructured carbon and its ability to interact with the polar constituents
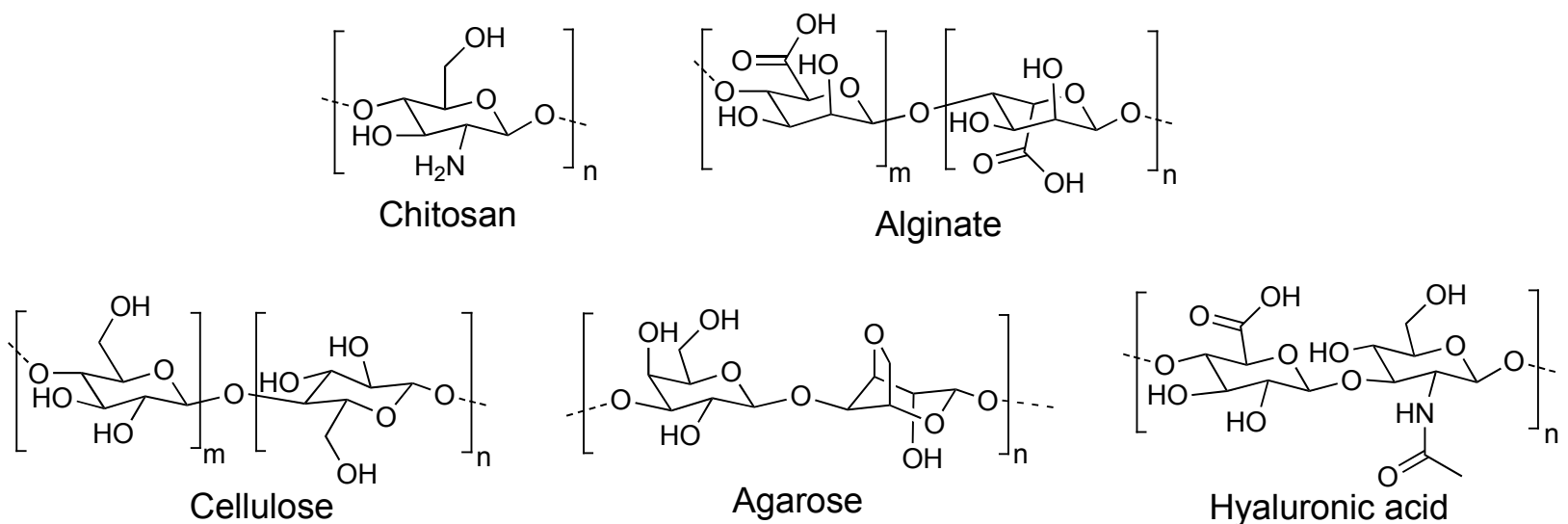

Fig. (5). Polysaccharides commonly used for hydrogels. 
of cells and culture medium is an important physicochemical factor that influences cell interactions and biological response. Furthermore, it is beneficial in promoting the biocompatibility and the degradation of the scaffold. The high water retention ability, the hydrophilic nature, and the interconnected porous structure of these scaffolds, facilitate cell attachment and proliferation, and enhance stability toward enzymatic degradation [54].

Carboxylated nanodiamonds have also been exploited to engineer chitosan-derived "smart" nanogels and achieve prolonged drug release. In particular, functionalized nanodiamonds are coated with polyethylene imine and then crosslinked with acetylated CS to form nanogels that can be loaded with drugs. Next, contact lenses can be embedded with the nanogels, and subsequent contact with lacrimal fluid leads to lysozyme-mediated CS degradation and nanogel disassembly, and thus, sustained drug delivery. Notably, the engineered contact lenses display enhanced mechanical properties, while maintaining optical clarity, water content and oxygen permeability [55].

In the case of graphene and GO as nanostructured carbon component, good dispersibility in water is easier to achieve. GO and CS can be used to form hybrid gels; additional CS functionalization with heparin improves hemocompatibility for use as absorbent for blood toxin removal. In this way, selective decontamination can be achieved, for instance by binding of bilirubin from blood samples [56]. Alternatively, reduced GO can also be well dispersed with CS, using lactic acid as a cross-linker to achieve a homogeneous composite with improved mechanical and conductive properties. The resulting hydrogel can be extrusion printed in $3 \mathrm{D}$ into scaffolds for tissue engineering, allowing infiltration of adherent cells (Fig. 6) [57].

CS porous scaffolds with GO $0.1 \mathrm{wt} \%$ and hydroxyapatite nanoparticles were found more bioactive than neat CS, as the release of $\mathrm{Ca}$ and $\mathrm{P}$ ions was increased after soaking the nanocomposites in stimulated body fluid [58]. Similarly, Li et al. prepared $\mathrm{CS} / \mathrm{GO}$ nanocomposites containing nanohydroxyapatite synthesized in situ, with improved mechanical properties [59]. The nanocomposites exhibited a high cell proliferation rate, and the $\mathrm{CS} / \mathrm{GO} /$ hydroxyapatite provided higher cell viability and alkaline phosphatase activity compared to the GO/hydroxyapatite nanocomposite.

Pinto et al. reviewed the biocompatibility of graphene nanocomposites and concluded that modification of GO with hydrophilic molecules or encapsulation in hydrophilic matrices improves cell adhesion and compatibility [60]. The addition of $3 \mathrm{wt} \% \mathrm{GO}$ in CS scaffolds improved the porosity by providing a better defined and well interconnected pore structure to the nanocomposites, without inducing significant cytotoxicity [61]. Cell attachment was found faster on graphene/CS nanocomposite films with graphene content $0.1-$ $0.3 \mathrm{wt} \%$, while displaying good biological safety and almost non-cytotoxicity [62].

CS-GO nanocomposite hydrogels find also applications in transdermal drug delivery as they allow for $\mathrm{pH}$-sensitive release [63]. Besides, graphene and fullerene are useful nanofillers to fine-tune the porosity of CS soft materials [64].

\subsection{Alginate Hydrogels}

Alginate is an anionic, water-insoluble polysaccharide; its sodium salt gellifies in the presence of calcium ions, and this is the main method used for gelation also in the presence of other components, such as CNTs or graphene. Alginate hydrogels are successfully reinforced by SWCNTs that have been previously dispersed by means of polymer wrapping [65]. Multinozzle biopolymer deposition is an ideal method to build composite alginate/SWCNT scaffolds that allow for cell attachment and proliferation [66]. GO can be incorporated in alginate hydrogel fibers also by wet spinning through a $\mathrm{CaCl}_{2}$ coagulating bath. The addition of $\mathrm{GO}$ significantly improves the strength of the fibers, which have similar water absorbency to alginate fibers in aqueous solution. Importantly, within a week of culture, rabbit transparent cartilage cells grow and spread on the gel [67].

Alginate-CNT conjugates can also be used for drug delivery in cancer therapy (Fig. 7). Oxidized SWCNTs can be wrapped by CS first, and alginate later, so that the two polysaccharides favourably interact with each other thanks to their opposed surface charges. The wrapped CNTs display improved solubility and stability in aqueous environments. Subsequent addition of doxorubicin, as cargo for the CNT, effectively yields an unconventional drug delivery system [68].

\subsection{Hyaluronic Acid Hydrogels}

Hyaluronic acid (HA) is an important component of extracellular matrices and is widely used as interface with biological systems for tissue engineering, wound healing or drug delivery [69].

Carboxylated SWCNTs can be efficiently dispersed in an aqueous solution of HA to yield a hybrid hydrogel by crosslinking with divinyl sulfone. While both the native and hybrid gels display shear-thinning behavior, the SWCNTcontaining gel has improved viscoelasticity, thanks to the participation of the hydroxyl functionality on the SWCNT surface, to the cross-linking of HA by divinyl sulfone. However, high water uptake capacity of the native gel and the hybrid gel are almost unchanged even after wt $\%$. SWCNT incorporation. Therefore, this kind of gels could find potential application in tissue engineering, due to unique properties in terms of water uptake capacity, and increased viscoelasticity [70]. CNT-HA conjugate systems are also promising as innovative therapeutic solutions for cancer. HA targets the receptor CD44 of gastric cancer stem cells, facilitating endocytosis of the CNTs and cell death [69].

Incorporation of GO in HA hydrogels is an interesting approach to increase the loading capacity towards hydrophobic drugs, such as doxorubicin [71]. HA-GO-Doxorubicin nanohybrid hydrogel was constructed by conjugating GO with $\mathrm{HA}$ via $\mathrm{H}$-bond interaction for targeted delivery of doxorubicin to induce selective cytotoxicity in target cancer cells [72]. The as prepared hydrogel possessed excellent physiological stability, high drug loading capacity for DOX, $\mathrm{pH}$ responsiveness, and it allowed sustained release of anticancer drugs selectively to target cells. This fact was demonstrated by efficient inhibition of proliferation only of target cells, without side effects to controls. Moreover, an in vivo 


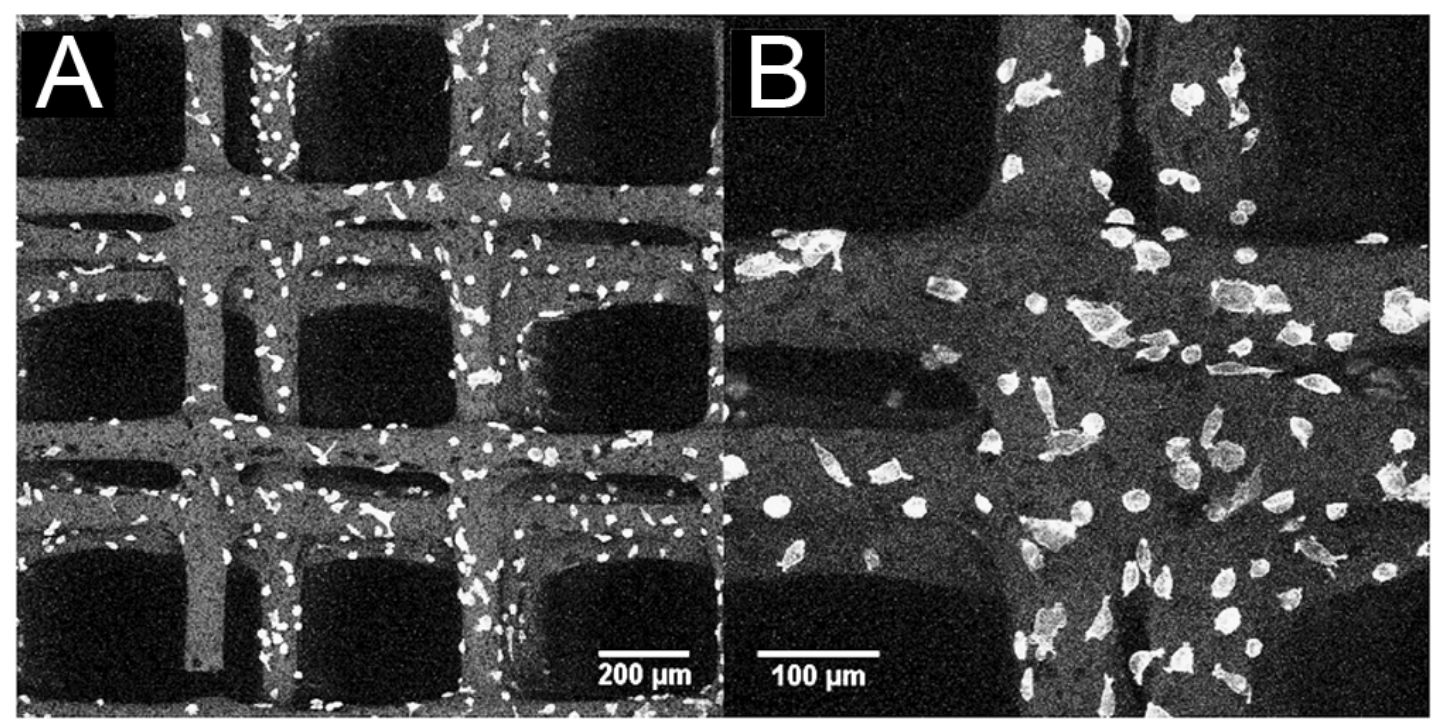

Fig. (6). Confocal microscopy stacked images at low (A) and high (B) magnification of reduced GO-CS composite hydrogel printed in 3D in a fibrous scaffold that allows adherent cell infiltration. Reprinted from Ref. [57], with permission of the Royal Society of Chemistry.

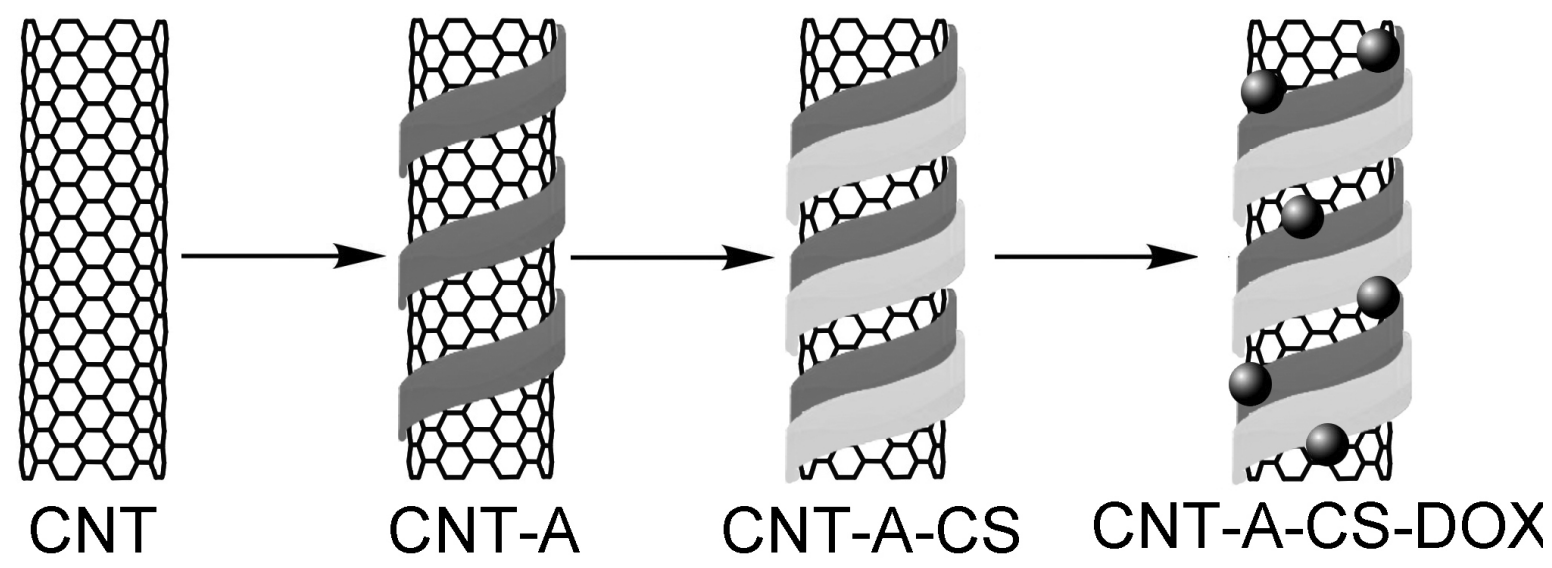

Fig. (7). TEM images (top) and scheme (bottom) showing the assembly of polysaccharide-CNT-drug conjugates for cancer therapy. From left to right: CNT alone, CNT wrapped with alginate (A), CNT wrapped with A and chitosan (CS), and finally CNT-A-CS with added doxorubicin (DOX). Adapted from ref. [68], Copyright (2009), with permission from Elsevier.

anticancer efficacy study using a mouse model demonstrated higher tumor inhibition rate of $\mathrm{HA}-\mathrm{GO}-\mathrm{DOX}$ compared to free DOX and GO-DOX formulation at an equivalent drug dose. Overall, GO-HA biomaterials could provide interesting therapeutic solutions, especially if taking advantage of GO unique properties that could allow for cancer cell photothermal ablation based on NIR-response [73].

\subsection{Cellulose Hydrogels}

The most abundant organic polymer on Earth, cellulose, is also a popular material for biomedical applications. Electrospun cellulose acetate nanofibers can be assembled with positively charged chitosan, and negatively charged MWCNTs or sodium alginate, via layer-by-layer technique. The incorporation of MWCNTs in the multi-layered fibrous scaffolds tends to improve attachment, spreading, and proliferation of mouse fibroblasts. With good protein adsorption capacity, mechanical durability, cell attachment and proliferation, and good hemocompatibility, the MWCNTcontaining multi-layered composites should find applications in tissue engineering and regenerative medicine. The facile assembly approach may be extended to immobilize also other bioactive components for scaffold tailoring to a range of biomedical uses [74].

Cellulose, and more recently nanocellulose, are also becoming popular building blocks to achieve biologically benign hydrogels; further addition of carbon nanostructures such as graphene and GO makes the composites electroactive, thus expanding the breadth of potential applications [75]. Cellulose and GO can also yield highly porous hydrogel monoliths characterised by high adsorbing capacity and compressive strength [76].

GO-bacterial cellulose nanocomposite hydrogels with well-dispersed GO are successfully developed using a facile one-step in situ biosynthesis by adding GO suspension into the culture medium of bacterial cellulose [77]. The nanocomposites exhibit significantly higher tensile strength and modulus over pristine cellulose, owing to the uniform dispersion and interaction between GO and cellulose. Such hydrogels are promising as tissue engineering scaffolds. 
Hyaluronic acid-fullerene conjugates have been proposed in photodynamic therapy, exploiting the $\mathrm{C}_{60}$ capability to generate ${ }^{1} \mathrm{O}_{2}$ upon photoirradiation. Various derivatives demonstrated good capability of inhibiting cell proliferation in HCT-116 or MCF7 cell cultures and consequent tumor regression in in vivo experiments [78].

\subsection{Agarose Hydrogels}

Agarose has the ability to disperse and accommodate CNTs, while maintaining thermo-responsive hydrogelation and the potential to be easily functionalized to introduce further chemical groups. Fibers containing the two components are rigid and tough when dry, but exhibit mechanical properties compliant with brain tissue once hydrated. They have shown to be not only non-toxic, but also biocompatible, and biologically modifiable. These properties, along with their stable electrical conductance, provide a novel material with high potential for future neurophysiologic use. The gelling properties of agarose allow it to be easily molded into various shapes with applications such as directed nerve repair and nerve guidance conduit [79].

Agarose is also capable of acting as stabilizer and physical cross-linker for graphene-based hydrogels, which display remarkable stability against a variety of harsh chemical conditions [80].

\subsection{Future Directions of Polysaccharide Hydrogels}

Polysaccharide hydrogels have been developed over the years for biomaterial applications. In particular, chitosan has shown to have the required physico-chemical and biological properties to become a popular choice that can be easily functionalized and adapted to various uses in medicine. In recent years, there has been the growing tendency to combine multiple components into scaffolds for improved performance, also using more than one polysaccharide altogether. Therefore, it is common to see chitosan in conjugation with other carbohydrates, such as alginate, or especially hyaluronic acid. The latter is indeed an elected choice for specific cell targeting, or, more generally, as external coating to interface with cells. In the case of hybrid or composite hydrogels, polysaccharides can also be combined with proteins to assist with carbon nanostructure dispersibility, and it is expected that this trend to use different components in sophisticated biomaterial designs will perdure and be further refined in the future. The combination of multiple gelling agents with carbon nanostructures is a useful approach to control the material, and interesting properties can be obtained, e.g., dual stimulus responsive drug release under the interaction of $\mathrm{pH}$ value and pulsatile electric field [81].

\section{SYNTHETIC POLYMER HYDROGELS}

\subsection{Acrylate Polymers}

Acrylate polymers were the first components used to obtain a hydrogel back in 1955 [4], and over the years they have been combined with other compounds, to achieve formulations with enhanced properties for use as biomaterials. For instance, methacrylate hydrogels can be combined with GO and sodium styrene sulfonate polymers to mimic the carboxylate and sulfonate functional groups of heparin, re- spectively (Fig. 8). The resulting materials display improved hemocompatibility, as expected from the heparin-mimicking function, revealing reduced platelet adhesion and anticoagulant property [82]. Their increased porosity and improved swelling ability allows for drug loading and subsequent prolonged release [83].

Alternatively, the conjugation of cross-linkable methacrylate functional groups with gelatin protein leads to a low-modulus, biodegradable GelMA hydrogel [84]. Various applications can be envisaged for this versatile polymer, also in combination with nanostructured carbon, e.g. to increase mechanical resilience and electrical conductivity. Cell encapsulation can be attained within carboxylated MWCNTGelMA microgels that can be photo-patterned [85]. A similar concept makes use of GO combined with GelMA [86]. Gene therapy is another possible application, upon embedding of polyethyleneimine-functionalized GO to form nanocomplexes with DNA. Inclusion of a pro-angiogenic VEGFencoding gene allows for transfection of myocardial tissue, and subsequent therapeutic effect on infarcted rat heart [87]. There are also other ways to achieve similar materials, for instance via functionalization of GO with methacrylate groups for subsequent copolymerization with GelMA into a soft material that allows fibroblast proliferation within a 7day period [88].

The (2-hydroxyethyl methacrylate) polymers, when prepared in the presence of fullerene, change their properties, since the swelling ratio that results at equilibrium appears to be lower than that of the pristine hydrogel. This behavior is not surprising considering the hydrophobic character of the embedded molecules. This variation can be modulated, by changing the fullerene percentage content in order to obtain matrices with fine-tuneable release rate of drugs [89]. If delivery of bioactive fullerenes is desired, this can be achieved from microgels via swelling and shear-induced stress [90].

Combination the acrylate derivative poly(acryloyl-6aminocaproic acid with GO offer the possibility to combine higher mechanical resilience of the gel with self-healing, a property that is highly sought after, especially in tissue regeneration [91]. Besides, addition of GO to pAA hydrogels offers the advantage of remarkably reducing the material brittleness [92]. Acrylate polymer composites bearing GO and antibacterial silver find applications in wound healing dressings [93]. When graphene is cross-linked to a poly(N,N-dimethylacrylamide) structure, the obtained hydrogel exhibits good neural compatibility, high conductivity, low impedance and efficient near-infrared-triggered photothermal self-healing behaviour [94].

Among acrylate polymers, poly( $N$-isopropylacrylamide) (pNIPAAm) has gained widespread popularity thanks to its thermoresponsive behavior (Fig. 9). When the polymer is anchored on a surface and immersed in an aqueous solution, at room temperature it is in its extended, hydrated "brush" form, while upon mild heating to $32{ }^{\circ} \mathrm{C}$ and over, it collapses to its shrunk, dehydrated "mushroom" form. Cells can attach to pNIPAAm at $37{ }^{\circ} \mathrm{C}$ and detach at $20{ }^{\circ} \mathrm{C}$ to form a continuous layer or "cell sheet" for tissue engineering. In contrast to the limited potential of the polymer alone, anchoragedependent "cell sheets" can be prepared from MWCNTinterpenetrated pNIPAAm hydrogels, thanks to the material 


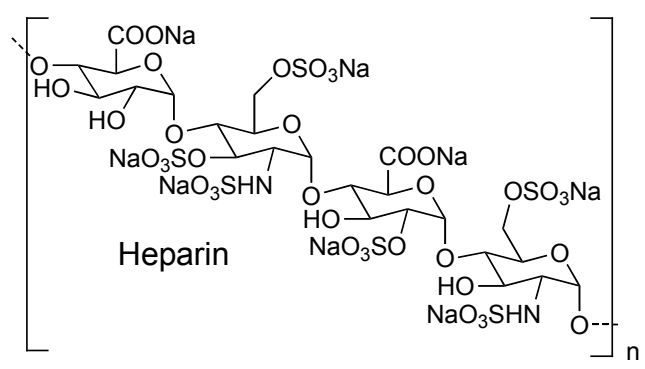

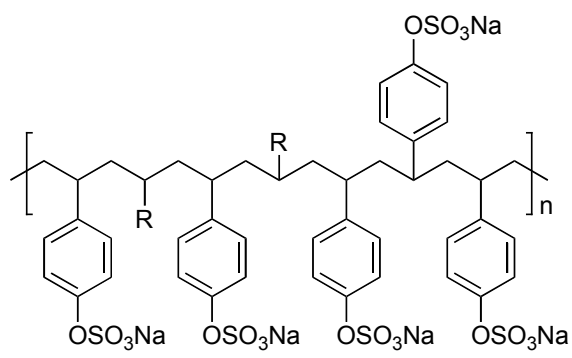

Sodium Styrene Sulfonate polymer

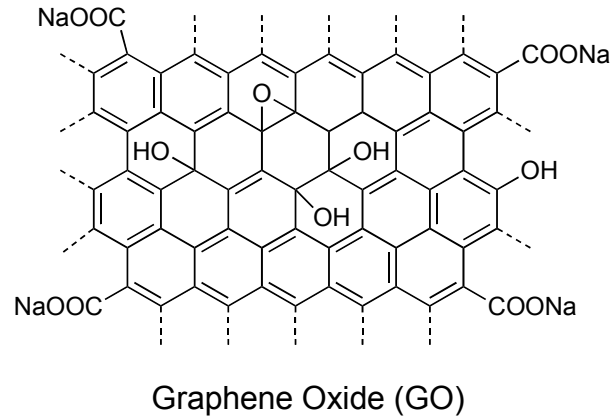

Graphene Oxide (GO)

Fig. (8). Heparin (top) sulfonate and carboxylate functional groups can be mimicked by a combination of sodium styrene sulfonate polymer (bottom left) and GO (bottom right), respectively [81].

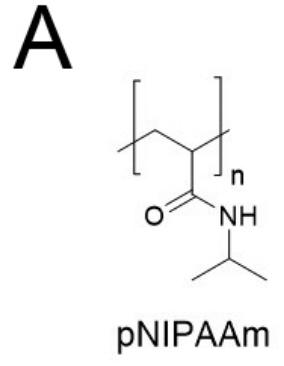

B

extended form "brush"

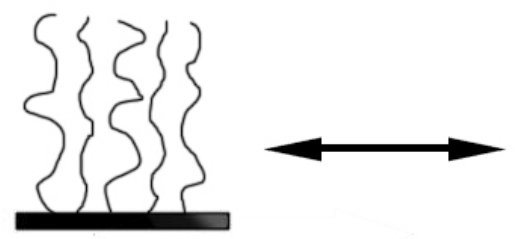

$\mathrm{T}=20^{\circ} \mathrm{C}$

\section{collapsed form "mushroom"}

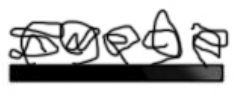

$\mathrm{T}=37{ }^{\circ} \mathrm{C}$

Fig. (9). A) pNIPAAm chemical structure. B) pNIPAAm thermo-responsive behavior in aqueous solutions at cell-culture relevant temperatures.

increased roughness and stiffness, allowing for appropriate cell spreading [95]. Similar effects are obtained with the incorporation of SWCNTs into pNIPAAm hydrogels [96]. Incorporation of GO into pNIPAAm hydrogels is also possible [97]. Alternatively, use of fullerene offers a means to tailor the material thermoresponse, by lowering the critical solution temperature thanks to the hydrophobic nature of $\mathrm{C}_{60}$ [98].

Beside the thermo-sensitive behavior, gels can be engineered also to respond to other external stimuli, thanks to the addition of the nanostructured carbon component. Incorporation of GO into a pNIPAAm hydrogel, previously functionalized with an RGD peptide to promote cell adhesion [99], is a useful means to obtain a gel that, upon near-infrared (NIR) irradiation, shrinks to release cells [100]. The nanocarbons allow for also other, important, added benefits in the case of injectable tissue engineering of conducting organs. For instance, engraftment and survival of stem cells is enhanced after their transplantation in infarcted myocardium, and even their therapeutic efficacy is improved [96]. Smart hydrogels can be engineered to respond to light also thanks to other photo-sensitive units. Host-guest supramolecular gels can be made by combining 1) a polysaccharide-cyclodextrin (i.e., the host) polymer used to wrap SWCNTs, thus favoring their dispersion in water, and 2) a pAA gelling polymer functionalized with a photo-responsive azobenzene moiety (i.e., the guest). Reversible gel/sol transition is achieved upon UV/Vis irradiation, thanks to cis/trans isomerization of the azobenzene group, which thus gets out/in the cyclodextrin, respectively [101].

Incorporation of nanocarbons is a useful approach to fine-tune a material electro-response. As an example, pulsatile drug delivery can be achieved by means of MWCNT [102] or graphene [103] incorporation into pMAA gels, and their subsequent de-swelling upon DC electric field stimulation. MWCNTs that are covalently incorporated in acrylatederived hydrogel films serve the dual function of both enhancing the electro-responsivity and modulating the release of anionic and cationic drugs (e.g., diclofenac sodium salt and ciprofloxacin) [104]. Polymer electro-response can be used also in tissue engineering. Dielectrophoretically aligned MWCNTs within a GelMA matrix offer a useful template 
for hierarchical alignment of myoblasts and myotubes, in order to maximize contractility and force generation within engineered muscle tissue [105]. MWCNT-GelMA hybrid hydrogels are useful for cardiac tissue engineering, thanks to MWCNT ability to form collagen fibril-like nanofibers bridging gel pores, thus improving cardiomyocyte electrical coupling and allowing for spontaneous synchronous beating of thin cardiac patches [106].

Another example of stimuli-responsive CNT-hydrogel composite is offered by using a polyvinylpyrrolidinium positively charged matrix wrapping on CNTs, the counter ion of which is a Europium complex, emitting at $614 \mathrm{~nm}$. In this case, the application of a magnetic field allows for the alignment of the tubes with a consequent anisotropic effect. Even though the biological application of this system is not straightforward, it may pave the way to the preparation of new theranostic devices [107].

\subsection{Poly(Vinyl Alcohol)}

Poly(vinyl alcohol) (PVA) hydrogels are distinguished by their ability to retain water in their structure very well, and thus maintain a moist environment for longer periods, relative to other synthetic hydrogels [4]. Introduction of rGO in a PVA matrix is a useful means to obtain electroresponsive hydrogels for pulsatile drug delivery in wound healing and transdermal therapy [108]. GO can also be crosslinked by using 2,2'-(ethylenedioxy)-diethanethiol prior to incorporation into the PVA matrix. The resulting composite hydrogels have improved mechanical properties and sustain chondrocyte survival and proliferation [109]. When GO is used as a nanofiller for PVA hydrogels via a freeze-thaw method, the resulting material displays increased tensile and compressive strength, while not showing cytotoxicity to osteoblasts in vitro [110].

\subsection{Poly(Ethylene Oxide)-Derived Polymers}

PEO, also known as poly(ethylene glycol) or PEG, is one of the most popular hydrogels for medicinal applications, thanks to its good biocompatibility that has been well-proven over the years in numerous examples [4]. Popular are also the derived PPO-copolymers, including the well-known Pluronics. Injectable hydrogels can be obtained from GOPluronic mixtures that undergo sol/gel transitions through physical cross-linking, induced in response to a variety of convenient stimuli, including NIR irradiation, mild heating, and $\mathrm{pH}$ change [111]. Pluronic also provides an effective means to chemically exfoliate graphene, with the hydrophobic PPO units interacalating between graphene sheets, and the hydrophilic PEO chains extending in the surrounding aqueous environment [112]. Thanks to graphene, the resulting hybrid hydrogel presents a lamellar structure that allows for shear-thinning behaviour, which, together with the reduced viscosity and thermo-response, makes these hybrids interesting injectable candidates for drug delivery [112]. A recent report highlighted the possibility to crosslink PEOPPO copolymers with GO by means of peroxidase catalysis, yielding injectable and mechanically robust hydrogels [113].

PEG-diacrylate hydrogels, embedding nanodiamonddaunorubicin complexes and temozolomide, demonstrate their capacity to slow down the release of antitumor drugs, avoiding the side effects due to massive and uncontrolled liberation of the active compounds, as it typically occurs during burst release. The prolonged delivery seems to be related to the presence of nanodiamonds, which provide a high surface for the loading of the drug. Moreover, the authors show how two-layered gel preparations can be designed, for the sequential availability of, first, temozolomide released from the PEG-hydrogel, and, later, daunorubicin released from the nanodiamond-complexes, for applications in combined therapy [114].

\subsection{Future Directions for Synthetic Polymer Hydrogels}

Among the many available options for synthetic polymer hydrogels, acrylate polymers have been probably the most studied, thanks to their efficient and well-known chemistry. However, the toxicity of acrylate monomers and of the other chemical components often used in the polymerization process is a high barrier for their effective implementation in novel solutions for medicinal use. By contrast, PEG derivatives have a more benign nature. They are already featured in many products currently present on the market, thus it is likely they will be receiving increasing attention for the development of hybrid or composite hydrogel biomaterials. Their conjugation with CNTs has already been widely exploited for medicinal applications, although not yet for the development of PEG-based scaffolds, but rather to simply improve CNT dispersibility in water for the use as drug vectors. Therefore, the development of PEG-based scaffolds containing CNTs or graphene derivatives seems to likely be the next step in this fast-moving research area.

\section{GRAPHENE HYDROGELS}

There is a growing interest in developing a variety of biomaterials based on graphene and/or GO building blocks, as their properties make them promising candidates for innovative applications in tissue engineering and bionics [115]. Moreover, graphene is a rising star also for drug delivery applications [116]. Although this 2D nanomaterial is a recent addition to the family of nanocarbons, its chemical modification is already quite well-established, both in dispersions [117] and on solid substrates [118], thus giving the opportunity to be easily manipulated for the creation of 3D biomaterials.

Self-supporting hydrogels can be achieved through the rational manipulation of the colloidal interactions between solvated graphene sheets, by means of physisorption of acrylamide polymer chains onto pristine graphene sheets without any binders or cross-linkers [119]. The resulting thin material has remarkable flexibility and adaptability [120]. Graphene-based hydrogels can be obtained via a hydrothermal route, and display remarkable conductivity, mechanical strength, thermal stability and high specific capacitance [121]. This approach effectively delivers a 3D nanostructured material in one step, through entrapment of water mediated by hydrogen bonding with oxygenated functional groups on GO and partial establishment of $\pi$-stacking interactions between GO sheets [121]. Supramolecular coassembly into hydrogels can also be obtained through electrostatic interactions between a positively charged drug (e.g., metformin hydrochloride), and the carboxylate groups of 
GO. Thanks to the cross-linking role played by the drug, the material displays $\mathrm{pH}$-dependent drug release profiles [122]. Alternatively, one-step fabrication of macroscopic hydrogel monoliths can be achieved by means of reduction of GO sheets by ferrous ions and in situ simultaneous deposition of iron-based nanoparticles on graphene sheets [123].

Preliminary studies over a 3-day period on GO hydrogel biocompatibility reveal a promising biomaterial performance as bone-derived cells are able to grow and protrude filopodia [124]. This kind of material has high potential for bone tissue regeneration, thanks to its ability to stimulate osteogenic differentiation of stem cells, and to an overall good biocompatibility profile upon subcutaneous implantation into rats [125].

It seems unlikely that graphene-only hydrogels will make their way into the clinic, but we cannot exclude that they may be further developed to optimize their use as scaffold skeleton, realizing their electro-responsive behavior to achieve "smart" engineered tissues or drug delivery systems.

\section{CONCLUSION}

In conclusion, we have seen how nanostructured carbon has already been integrated with a wide variety of hydrogels for biomaterial applications, spanning from protein/peptides, through polysaccharides to synthetic polymers, for tissue engineering and on-demand drug release. The vast majority of these studies are still tackling basic research questions in vitro, with only a few attempting to address the issues associated with more complex research models in vivo. Clearly, this is still a young, emerging area that poses many challenges to be addressed, for its realistic implementation into therapy. However, more and more medicinal chemists are being fascinated by the unique properties of nanocarbons, and it is only through the determined research efforts to make them more biocompatible, that novel therapeutic paradigms can be realized to find credible solutions to unresolved therapeutic dilemmas.

\section{LIST OF ABBREVIATIONS}

$\begin{array}{ll}\mathrm{CNT} & =\text { carbon nanotube } \\ \mathrm{CS} & =\text { chitosan } \\ \mathrm{DC} & =\text { direct current } \\ \text { Fmoc } & =\text { fluorenylmethyloxycarbonyl } \\ \mathrm{GelMA} & =\text { gelatin methacrylate } \\ \mathrm{GO} & =\text { graphene oxide } \\ \mathrm{GSH} & =\text { glutathione } \\ \mathrm{MWCNT} & =\text { multi-wall carbon nanotube } \\ \text { pAA } & =\text { poly(acrylic acid) } \\ \mathrm{PEO} & =\text { poly(ethylene) oxide } \\ \mathrm{Phe} & =\text { Phenylalanine } \\ \text { pMAA } & =\text { poly(methacrylic acid }) \\ \text { pNIPAAm } & =\text { poly }(N \text {-isopropylacrylamide) } \\ \text { PPO } & =\text { poly(propylene) oxide }\end{array}$
65 (15), 1899-1920. applications. Chem. Chem. Technol. 2010, 4, 297-304.

[5]. Marchesan, S.; Prato, M., Nanomaterials for (nano)medicine. ACS Med. Chem. Lett. 2013, 4 (2), 147-149.

[6]. Ku, S. H.; Lee, M.; Park, C. B., Carbon-based nanomaterials for tissue engineering. Adv. Healthc. Mater. 2013, 2 (2), 244-260 Marchesan, S.; Melchionna, M.; Prato, M., Carbon nanostructures for nanomedicine: Opportunities and challenges. Fullerenes, Nanotubes Carbon Nanostruct. 2013, 22 (1-3), 190-195.

[7]. Ali-Boucetta, H.; Nunes, A.; Sainz, R.; Herrero, M. A.; Tian, B.; Prato, M.; Bianco, A.; Kostarelos, K., Asbestos-like pathogenicity of long carbon nanotubes alleviated by chemical functionalization. Angew. Chem. Int. Ed. 2013, 52 (8), 2274-2278.

[8]. Marchesan, S.; Kostarelos, K.; Bianco, A.; Prato, M., The winding road for carbon nanotubes in nanomedicine. Mater. Today 2015, 18 (1), 12-19.

[9]. Battigelli, A.; Menard-Moyon, C.; Da Ros, T.; Prato, M.; Bianco, A., Endowing carbon nanotubes with biological and biomedical properties by chemical modifications. Adv. Drug Deliv. Rev. 2013,

Rivero, R.; Grosso, C.; Motta, C.; Barbero, C.; Vivas, A., In vitro toxicity evaluation of hydrogel-carbon nanotubes composites on intestinal cells. J. Appl. Polym. Sci. 2015, 132 (5), 41370, doi: 10.1002/app.41370.

[11]. Saito, N.; Haniu, H.; Usui, Y.; Aoki, K.; Hara, K.; Takanashi, S.; Shimizu, M.; Narita, N.; Okamoto, M.; Kobayashi, S.; Nomura, H.; Kato, H.; Nishimura, N.; Taruta, S.; Endo, M., Safe clinical use of carbon nanotubes as innovative biomaterials. Chem. Rev. 2014, 114 (11), 6040-6079

[12]. Mulvey, J. J.; Villa, C. H.; McDevitt, M. R.; Escorcia, F. E.; Casey, E.; Scheinberg, D. A., Self-assembly of carbon nanotubes and antibodies on tumours for targeted amplified delivery. Nat. Nanotechnol. 2013, 8 (10), 763-771

[13]. Chu, T.-W.; Kopecek, J., Drug-free macromolecular therapeutics a new paradigm in polymeric nanomedicines. Biomater. Sci. 2015 3 (7), 908-922

[14]. Marchesan, S.; Prato, M., Under the lens: Carbon nanotube and protein interaction at the nanoscale. Chem. Commun. 2015, 51, $4347-4359$.

[15]. Voge, C. M.; Kariolis, M.; MacDonald, R. A.; Stegemann, J. P., Directional conductivity in SWNT-collagen-fibrin composite biomaterials through strain-induced matrix alignment. J. Biomed. Mater. Res. A 2008, 86 (1), 269-277; MacDonald, R. A.; Voge, C. M.; Kariolis, M.; Stegemann, J. P., Carbon nanotubes increase the elec- 
trical conductivity of fibroblast-seeded collagen hydrogels. Acta Biomater. 2008, 4 (6), 1583-1592.

[16]. Li, C.; Mezzenga, R., Functionalization of multiwalled carbon nanotubes and their $\mathrm{pH}$-responsive hydrogels with amyloid fibrils. Langmuir 2012, 28 (27), 10142-10146.

[17]. Li, C.; Mezzenga, R., The interplay between carbon nanomaterials and amyloid fibrils in bio-nanotechnology. Nanoscale 2013, 5 (14), 6207-6218.

[18]. Huang, L.; Li, C.; Yuan, W.; Shi, G., Strong composite films with layered structures prepared by casting silk fibroin-graphene oxide hydrogels. Nanoscale 2013, 5 (9), 3780-3786.

[19]. Zhou, J.; Chen, J.; Sun, H.; Qiu, X.; Mou, Y.; Liu, Z.; Zhao, Y.; Li, X.; Han, Y.; Duan, C.; Tang, R.; Wang, C.; Zhong, W.; Liu, J.; Luo, Y.; Xing, M.; Wang, C., Engineering the heart: Evaluation of conductive nanomaterials for improving implant integration and cardiac function. Sci. Rep. 2014, 4, Art. ID 3733, doi:10.1038/srep03733.

[20]. Paul, A.; Shao, W.; Shum-Tim, D.; Prakash, S., The attenuation of restenosis following arterial gene transfer using carbon nanotube coated stent incorporating tat/DNA(ang1+vegf) nanoparticles. Biomaterials 2012, 33 (30), 7655-7664.

[21]. Zhuang, Y.; Yu, F.; Ma, J.; Chen, J., Adsorption of ciprofloxacin onto graphene-soy protein biocomposites. New J. Chem. 2015, 39 (5), 3333-3336.

[22]. Song, Y.; Zhang, M.; Zhao, L.; Yin, X.; Zhao, J.; Li, J.; He, R.; Chang, Y.; Jin, J.; Zhao, Y.; Li, J.; Xing, G., Regulation on mechanical properties of collagen: Enhanced bioactivities of metallofullerol. Nanomed.-Nanotechnol. 2014, 10 (4), 783-793.

[23]. Sheikholeslam, M.; Pritzker, M.; Chen, P., Hybrid peptide-carbon nanotube dispersions and hydrogels. Carbon 2014, 71 (0), 284-293.

[24]. Wu, J.; Chen, A.; Qin, M.; Huang, R.; Zhang, G.; Xue, B.; Wei, J.; Li, Y.; Cao, Y.; Wang, W., Hierarchical construction of a mechanically stable peptide-graphene oxide hybrid hydrogel for drug delivery and pulsatile triggered release in vivo. Nanoscale 2015, 7 (5), 1655-1660.

[25]. MacEwan, S. R.; Chilkoti, A., Applications of elastin-like polypeptides in drug delivery. J. Control. Release 2014, 190, 314-330.

[26]. Wang, E.; Desai, M. S.; Heo, K.; Lee, S.-W., Graphene-based materials functionalized with elastin-like polypeptides. Langmuir 2014, 30 (8), 2223-2229.

[27]. Bartocci, S.; Mazzier, D.; Moretto, A.; Mba, M., A peptide topological template for the dispersion of [60]fullerene in water. Org. Biomol. Chem. 2015, 13 (2), 348-352.

[28]. Adams, D. J., Dipeptide and tripeptide conjugates as lowmolecular-weight hydrogelators. Macromol. Biosci. 2011, 11 (2), $160-173$.

[29]. Marchesan, S.; Waddington, L.; Easton, C.; Kushkaki, F.; McLean, K.; Forsythe, J.; Hartley, P., Tripeptide self-assembled hydrogels: Soft nanomaterials for biological applications. BioNanoSci. 2013, 3 (1), 21-29; Marchesan, S.; Qu, Y.; Waddington, L. J.; Easton, C. D.; Glattauer, V.; Lithgow, T. J.; McLean, K. M.; Forsythe, J. S.; Hartley, P. G., Self-assembly of ciprofloxacin and a tripeptide into an antimicrobial nanostructured hydrogel. Biomaterials 2013, 34 (14), 3678-3687.

[30]. Marchesan, S., Recent patents on peptide self-assembled hydrogels as nanostructured biomaterials. Recent Pat. Nanomed. 2014, 4 (2), 77-81.

[31]. Roy, S.; Banerjee, A., Functionalized single walled carbon nanotube containing amino acid based hydrogel: A hybrid nanomaterial. RSC Adv. 2012, 2 (5), 2105-2111.

[32]. Adhikari, B.; Banerjee, A., Short peptide based hydrogels: Incorporation of graphene into the hydrogel. Soft Matter 2011, 7 (19), 9259-9266.

[33]. Mba, M.; Jiménez, A. I.; Moretto, A., Templating the self-assembly of pristine carbon nanostructures in water. Chem. - Eur. J. 2014, 20 (14), 3888-3893

[34]. Yang, Q.; Wang, Z.; Weng, J., Self-assembly of natural tripeptide glutathione triggered by graphene oxide. Soft Matter 2012, 8 (38), 9855-9863.

[35]. Mo, Y.; Wang, H.; Liu, J.; Lan, Y.; Guo, R.; Zhang, Y.; Xue, W.; Zhang, Y., Controlled release and targeted delivery to cancer cells of doxorubicin from polysaccharide-functionalised single-walled carbon nanotubes. J. Mat. Chem. B 2015, 3 (9), 1846-1855.

[36]. Martins, A. M.; Eng, G.; Caridade, S. G.; Mano, J. F.; Reis, R. L.; Vunjak-Novakovic, G., Electrically conductive chitosan/carbon scaffolds for cardiac tissue engineering. Biomacromolecules 2014, 15 (2), 635-643.

[37]. Tercero, J. E.; Namin, S.; Lahiri, D.; Balani, K.; Tsoukias, N.; Agarwal, A., Effect of carbon nanotube and aluminum oxide addition on plasma-sprayed hydroxyapatite coating's mechanical properties and biocompatibility. Mater. Sci. Eng. C 2009, 29 (7), 2195 2202

[38]. Stankovich, S.; Dikin, D. A.; Dommett, G. H. B.; Kohlhaas, K. M.; Zimney, E. J.; Stach, E. A.; Piner, R. D.; Nguyen, S. T.; Ruoff, R. S., Graphene-based composite materials. Nat. 2006, 442 (7100), 282-286.

[39]. Zhang, H.; Zhou, L.; Zhang, W., Control of scaffold degradation in tissue engineering: A review. Tissue Eng. Part B 2014, 20 (5), 492 502.

[40]. Carson, L.; Kelly-Brown, C.; Stewart, M.; Oki, A.; Regisford, G.; Luo, Z.; Bakhmutov, V. I., Synthesis and characterization of chitosan-carbon nanotube composites. Mater. Lett. 2009, 63 (6-7), $617-$ 620 .

[41]. Jayakumar, R.; Menon, D.; Manzoor, K.; Nair, S. V.; Tamura, H., Biomedical applications of chitin and chitosan based nanomaterials - a short review. Carbohydr. Polym. 2010, 82 (2), 227-232.

[42]. Yadav, S. K.; Jung, Y. C.; Yang, C.-M.; Ko, Y.-I.; Yang, K.-S.; Kim, Y. A.; Cho, J. W., An environmentally friendly approach to functionalizing carbon nanotubes for fabricating a strong biocomposite film. RSC Adv. 2014, 4 (11), 5382-5388.

[43]. Venkatesan, J.; Kim, S.-K., Chitosan composites for bone tissue engineering —an overview. Mar. Drugs 2010, 8 (8), 2252-2266.

[44]. Huang, Y.-C.; Hsu, S.-H.; Kuo, W.-C.; Chang-Chien, C.-L.; Cheng, H.; Huang, Y.-Y., Effects of laminin-coated carbon nanotube/chitosan fibers on guided neurite growth. J. Biomed. Mater. Res. A 2011, $99 A$ (1), 86-93.

[45]. Venkatesan, J.; Qian, Z.-J.; Ryu, B.; Ashok Kumar, N.; Kim, S.-K. Preparation and characterization of carbon nanotube-graftedchitosan - natural hydroxyapatite composite for bone tissue engineering. Carbohydr. Polym. 2011, 83 (2), 569-577.

[46]. Wang, S.-F.; Shen, L.; Zhang, W.-D.; Tong, Y.-J., Preparation and mechanical properties of chitosan/carbon nanotubes composites. Biomacromolecules 2005, 6 (6), 3067-3072.

[47]. Lau, C.; Cooney, M. J.; Atanassov, P., Conductive macroporous composite chitosan-carbon nanotube scaffolds. Langmuir 2008, 24 (13), 7004-7010.

[48]. Patel, K. D.; Kim, T.-H.; Lee, E.-J.; Han, C.-M.; Lee, J.-Y.; Singh, R. K.; Kim, H.-W., Nanostructured biointerfacing of metals with carbon nanotube/chitosan hybrids by electrodeposition for cell stimulation and therapeutics delivery. ACS Appl. Mater. Interfaces 2014, 6 (22), 20214-20224.

[49]. Kumar, B.; Feller, J.-F.; Castro, M.; Lu, J., Conductive biopolymer nano-composites (CPC): Chitosan-carbon nanotube transducers assembled via spray layer-by-layer for volatile organic compound sensing. Talanta 2010, 81 (3), 908-915.

[50]. Sukrut, O.; Manjeet, J.; Ashwini, K. A., $\mathrm{pH}$ and electrical actuation of single walled carbon nanotube/chitosan composite fibers. Smart Mater. Struct. 2008, 17 (5), 055016

[51]. Spinks, G. M.; Shin, S. R.; Wallace, G. G.; Whitten, P. G.; Kim, S. I.; Kim, S. J., Mechanical properties of chitosan/CNT microfibers obtained with improved dispersion. Sensors Actuat. B-Chem. 2006, 115 (2), 678-684.

[52]. Li, C.; Yang, K.; Zhang, Y.; Tang, H.; Yan, F.; Tan, L.; Xie, Q.; Yao, S., Highly biocompatible multi-walled carbon nanotubechitosan nanoparticle hybrids as protein carriers. Acta Biomater. 2011, 7 (8), 3070-3077.

[53]. Pok, S.; Vitale, F.; Eichmann, S. L.; Benavides, O. M.; Pasquali, M.; Jacot, J. G., Biocompatible carbon nanotube-chitosan scaffold matching the electrical conductivity of the heart. ACS Nano 2014, 8 (10), 9822-9832.

[54]. Depan, D.; Misra, R. D. K., Processing-structure-functional property relationship in organic-inorganic nanostructured scaffolds for bone-tissue engineering: The response of preosteoblasts. J. Biomed. Mater. Res. A 2012, 100 A (11), 3080-3091.

[55]. Kim, H.-J.; Zhang, K.; Moore, L.; Ho, D., Diamond nanogelembedded contact lenses mediate lysozyme-dependent therapeutic release. ACS Nano 2014, 8 (3), 2998-3005.

[56]. Wei, H.; Han, L.; Tang, Y.; Ren, J.; Zhao, Z.; Jia, L., Highly flexible heparin-modified chitosan/graphene oxide hybrid hydrogel as a super bilirubin adsorbent with excellent hemocompatibility. J. Mat. Chem. B 2015, 3, 1646-1654. 
[57]. Sayyar, S.; Murray, E.; Thompson, B. C.; Chung, J.; Officer, D. L.; Gambhir, S.; Spinks, G. M.; Wallace, G. G., Processable conducting graphene/chitosan hydrogels for tissue engineering. J. Mat. Chem. B 2015, 3 (3), 481-490.

[58]. Mohandes, F.; Salavati-Niasari, M., Freeze-drying synthesis, characterization and in vitro bioactivity of chitosan/graphene oxide/hydroxyapatite nanocomposite. RSC Adv. 2014, 4 (49), 2599326001 .

[59]. Li, M.; Wang, Y.; Liu, Q.; Li, Q.; Cheng, Y.; Zheng, Y.; Xi, T.; Wei, S., In situ synthesis and biocompatibility of nano hydroxyapatite on pristine and chitosan functionalized graphene oxide. J. Mater. Chem. B 2013, 1 (4), 475-484.

[60]. Pinto, A. M.; Gonçalves, I. C.; Magalhães, F. D., Graphene-based materials biocompatibility: A review. Coll. Surf. B 2013, 111, 188202.

[61]. Dinescu, S.; Ionita, M.; Pandele, A. M.; Galateanu, B.; Iovu, H.; Ardelean, A.; Costache, M.; Hermenean, A., In vitro cytocompatibility evaluation of chitosan/graphene oxide 3D scaffold composites designed for bone tissue engineering. Biomed. Mater. Eng. 2014, 24 (6), 2249-2256.

[62]. Fan, H.; Wang, L.; Zhao, K.; Li, N.; Shi, Z.; Ge, Z.; Jin, Z., Fabrication, mechanical properties, and biocompatibility of graphenereinforced chitosan composites. Biomacromolecules 2010, 11 (9), 2345-2351.

[63]. Justin, R.; Chen, B., Characterisation and drug release performance of biodegradable chitosan-graphene oxide nanocomposites. Carbohydr. Polym. 2014, 103, 70-80.

[64]. Fahim, I. S.; Marei, N.; Salem, H. G.; Mamdouh, W., Effect of graphene and fullerene nanofillers on controlling the pore size and physicochemical properties of chitosan nanocomposite mesoporous membranes. J. Nanomater. 2015, 2015, Article ID 979561, doi:10.1155/2015/979561

[65]. Yan, L. Y.; Chen, H.; Li, P.; Kim, D. H.; Chan-Park, M. B., Finely dispersed single-walled carbon nanotubes for polysaccharide hydrogels. ACS Appl. Mater. Interfaces 2012, 4 (9), 4610-4615.

[66]. Venkatesan, J.; Pallela, R.; Kim, S.-K., Dispersion of single walled carbon nanotubes in marine polysaccharides for bone tissue engineering. J. Biomater. Tissue Eng. 2014, 4 (6), 501-505.

[67]. He, Y.; Zhang, N.; Gong, Q.; Qiu, H.; Wang, W.; Liu, Y.; Gao, J., Alginate/graphene oxide fibers with enhanced mechanical strength prepared by wet spinning. Carbohydr. Polym. 2012, 88 (3), 11001108.

[68]. Zhang, X.; Meng, L.; Lu, Q.; Fei, Z.; Dyson, P. J., Targeted delivery and controlled release of doxorubicin to cancer cells using modified single wall carbon nanotubes. Biomaterials 2009, 30 (30), 6041-6047.

[69]. Yao, H. J.; Zhang, Y. G.; Sun, L.; Liu, Y., The effect of hyaluronic acid functionalized carbon nanotubes loaded with salinomycin on gastric cancer stem cells. Biomaterials 2014, 35 (33), 9208-9223.

[70]. Bhattacharyya, S.; Guillot, S.; Dabboue, H.; Tranchant, J.-F.; Salvetat, J.-P., Carbon nanotubes as structural nanofibers for hyaluronic acid hydrogel scaffolds. Biomacromolecules 2008, 9 (2), 505-509.

[71]. Byun, E.; Lee, H., Enhanced loading efficiency and sustained release of doxorubicin from hyaluronic acid/graphene oxide composite hydrogels by a mussel-inspired catecholamine. J. Nanosci. Nanotechnol. 2014, 14 (10), 7395-7401.

[72]. Song, E.; Han, W.; Li, C.; Cheng, D.; Li, L.; Liu, L.; Zhu, G.; Song, Y.; Tan, W., Hyaluronic acid-decorated graphene oxide nanohybrids as nanocarriers for targeted and ph-responsive anticancer drug delivery. ACS Appl. Mater. Interfaces 2014, 6 (15), 11882-11890.

[73]. Jung, H. S.; Kong, W. H.; Sung, D. K.; Lee, M.-Y.; Beack, S. E.; Keum, D. H.; Kim, K. S.; Yun, S. H.; Hahn, S. K., Nanographene oxide-hyaluronic acid conjugate for photothermal ablation therapy of skin cancer. ACS Nano 2014, 8 (1), 260-268.

[74]. Luo, Y.; Wang, S.; Shen, M.; Qi, R.; Fang, Y.; Guo, R.; Cai, H.; Cao, X.; Tomás, H.; Zhu, M.; Shi, X., Carbon nanotubeincorporated multilayered cellulose acetate nanofibers for tissue engineering applications. Carbohydr. Polym. 2013, 91 (1), 419427.

[75]. Shi, Z.; Phillips, G. O.; Yang, G., Nanocellulose electroconductive composites. Nanoscale 2013, 5 (8), 3194-3201.

[76]. Zhang, Y.; Liu, Y.; Wang, X.; Sun, Z.; Ma, J.; Wu, T.; Xing, F.; Gao, J., Porous graphene oxide/carboxymethyl cellulose monoliths, with high metal ion adsorption. Carbohydr. Polym. 2014, 101, 392400.

[77]. Si, H.; Luo, H.; Xiong, G.; Yang, Z.; Raman, S. R.; Guo, R.; Wan, Y., One-step in situ biosynthesis of graphene oxide-bacterial cellulose nanocomposite hydrogels. Macromol. Rapid Commun. 2014, 35 (19), 1706-1711.

[78]. Kwag, D.S.; Park, K.; Oh, K.T.; Lee, E.S., Hyaluronated fullerenes with photoluminescent and antitumoral activity. Chem. Commun. 2013, 49 (3), 282-284; Kim, S.; Park, J.; Youn, Y.S.; Oh, K.T.; Bae, J.H.; Lee, E.S., Hoechst 33258-conjugated hyaluronated fullerene for efficient photodynamic tumor therapy and necrotic tumor targeting. J. Bioact. Compat. Pol. 2015, 30 (3), 275-288; Zhang, H.; Hou, L.; Jiao, X.; Ji, Y.; Zhu, X.; Zhang, Z., Transferrin-mediated fullerenes nanoparticles as $\mathrm{Fe}^{2+}$-dependent drug vehicles for synergistic anti-tumor efficacy Biomaterials 2015, 37, 353366.

[79]. Lewitus, D. Y.; Landers, J.; Branch, J.; Smith, K. L.; Callegari, G.; Kohn, J.; Neimark, A. V., Biohybrid carbon nanotube/agarose fibers for neural tissue engineering. Adv. Funct. Mater. 2011, 21 (14), 2624-2632.

[80]. Wang, Y.; Zhang, P.; Liu, C. F.; Huang, C. Z., A facile and green method to fabricate graphene-based multifunctional hydrogels for miniature-scale water purification. RSC Adv. 2013, 3 (24), 92409246

[81]. Shi, X.; Zheng, Y.; Wang, C.; Yue, L.; Qiao, K.; Wang, G.; Wang, L.; Quan, H., Dual stimulus responsive drug release under the interaction of $\mathrm{pH}$ value and pulsatile electric field for a bacterial cellulose/sodium alginate/multi-walled carbon nanotube hybrid hydrogel. RSC Adv. 2015, 5 (52), 41820-41829.

[82]. He, C.; Shi, Z.-Q.; Ma, L.; Cheng, C.; Nie, C.-X.; Zhou, M.; Zhao, C.-S., Graphene oxide based heparin-mimicking and hemocompatible polymeric hydrogels for versatile biomedical applications. J. Mat. Chem. B 2015, 3 (4), 592-602.

[83]. He, C.; Shi, Z.-Q.; Ma, L.; Cheng, C.; Nie, C.-X.; Zhou, M.; Zhao, C.-S., Graphene oxide based heparin-mimicking and hemocompatible polymeric hydrogels for versatile biomedical applications. J. Mater. Chem. B 2015, 3 (4), 592-602.

[84]. Nichol, J. W.; Koshy, S. T.; Bae, H.; Hwang, C. M.; Yamanlar, S.; Khademhosseini, A., Cell-laden microengineered gelatin methacrylate hydrogels. Biomaterials 2010, 31 (21), 5536-5544.

[85]. Shin, S. R.; Bae, H.; Cha, J. M.; Mun, J. Y.; Chen, Y. C.; Tekin, H.; Shin, H.; Farshchi, S.; Dokmeci, M. R.; Tang, S.; Khademhosseini, A., Carbon nanotube reinforced hybrid microgels as scaffold materials for cell encapsulation. ACS Nano 2012, 6 (1), 362372.

[86]. Shin, S. R.; Aghaei-Ghareh-Bolagh, B.; Dang, T. T.; Topkaya, S. N.; Gao, X.; Yang, S. Y.; Jung, S. M.; Oh, J. H.; Dokmeci, M. R.; Tang, X. S., Cell-laden microengineered and mechanically tunable hybrid hydrogels of gelatin and graphene oxide. Adv. Mater. 2013, 25 (44), 6385-6391.

[87]. Paul, A.; Hasan, A.; Kindi, H. A.; Gaharwar, A. K.; Rao, V. T. S.; Nikkhah, M.; Shin, S. R.; Krafft, D.; Dokmeci, M. R.; Shum-Tim, D.; Khademhosseini, A., Injectable graphene oxide/hydrogel-based angiogenic gene delivery system for vasculogenesis and cardiac repair. ACS Nano 2014, 8 (8), 8050-8062.

[88]. Cha, C.; Shin, S. R.; Gao, X.; Annabi, N.; Dokmeci, M. R.; Tang, $\mathrm{X}$.; Khademhosseini, A., Controlling mechanical properties of cellladen hydrogels by covalent incorporation of graphene oxide. Small 2014, $10(3), 514-523$.

[89]. Katiyar R.; Bag D. S.; Nigam, I., Synthesis and evaluation of swelling characteristics of fullerene $\left(\mathrm{C}_{60}\right)$ containing cross-linked poly(2-hydroxyethyl methacrylate) hydrogels. Adv. Mat. Lett. 2014, 5 (4), 214-222.

[90]. Tarabukina, E.; Zoolshoev, Z.; Melenevskaya, E.; Budtova, T., Delivery of fullerene-containing complexes via microgel swelling and shear-induced release. Int. J. Pharm. 2010, 384 (1-2), 9-14.

[91]. Cong, H.-P.; Wang, P.; Yu, S.-H., Stretchable and self-healing graphene oxide-polymer composite hydrogels: A dual-network design. Chem. Mater. 2013, 25 (16), 3357-3362.

[92]. Shen, J.; Yan, B.; Li, T.; Long, Y.; Li, N.; Ye, M., Mechanical, thermal and swelling properties of poly(acrylic acid)-graphene oxide composite hydrogels. Soft Matter 2012, 8 (6), 1831-1836.

[93]. Fan, Z.; Liu, B.; Wang, J.; Zhang, S.; Lin, Q.; Gong, P.; Ma, L.; Yang, S., A novel wound dressing based on ag/graphene polymer hydrogel: Effectively kill bacteria and accelerate wound healing. Adv. Funct. Mater. 2014, 24 (25), 3933-3943. 
[94]. Hou, C.; Duan, Y.; Zhang, Q.; Wang, H.; Li, Y., Bio-applicable and electroactive near-infrared laser-triggered self-healing hydrogels based on graphene networks. J. Mat. Chem. 2012, 22 (30), 14991-14996.

[95]. Chen, Y. S.; Tsou, P. C.; Lo, J. M.; Tsai, H. C.; Wang, Y. Z.; Hsiue, G. H., Poly(n-isopropylacrylamide) hydrogels with interpenetrating multiwalled carbon nanotubes for cell sheet engineering. Biomaterials 2013, 34 (30), 7328-7334.

[96]. Li, X.; Zhou, J.; Liu, Z.; Chen, J.; Lu, S.; Sun, H.; Li, J.; Lin, Q.; Yang, B.; Duan, C.; Xing, M. M.; Wang, C., A PNIPAAM-based thermosensitive hydrogel containing SWCNTS for stem cell transplantation in myocardial repair. Biomaterials 2014, 35 (22), 56795688.

[97]. Zhang, E.; Wang, T.; Lian, C.; Sun, W.; Liu, X.; Tong, Z., Robust and thermo-response graphene-PNIPAM hybrid hydrogels reinforced by hectorite clay. Carbon 2013, 62, 117-126.

[98]. Katiyar, R.; Bag, D. S.; Nigam, I., Fullerene $\left(\mathrm{C}_{60}\right)$ containing poly (n-isopropylacrylamide) thermo-responsive smart hydrogels and their swelling behavior. J. Polym. Mater. 2013, 30 (1), 15.

[99]. Gahmberg, C. G.; Fagerholm, S. C.; Nurmi, S. M.; Chavakis, T.; Marchesan, S.; Gronholm, M., Regulation of integrin activity and signalling. Biochim. Biophys. Acta 2009, 1790 (6), 431-444.

[100]. Li, W.; Wang, J.; Ren, J.; Qu, X., 3d graphene oxide-polymer hydrogel: Near-infrared light-triggered active scaffold for reversible cell capture and on-demand release. Adv. Mater. 2013, 25 (46), 6737-6743.

[101]. Tamesue, S.; Takashima, Y.; Yamaguchi, H.; Shinkai, S.; Harada, A., Photochemically controlled supramolecular curdlan/singlewalled carbon nanotube composite gel: Preparation of molecular distaff by cyclodextrin modified curdlan and phase transition control. Eur. J. Org. Chem. 2011, 2011 (15), 2801-2806.

[102]. Servant, A.; Bussy, C.; Al-Jamal, K.; Kostarelos, K., Design, engineering and structural integrity of electro-responsive carbon nanotube- based hydrogels for pulsatile drug release. J. Mat. Chem. B 2013, 1 (36), 4593-4600.

[103]. Servant, A.; Leon, V.; Jasim, D.; Methven, L.; Limousin, P.; Fernandez-Pacheco, E. V.; Prato, M.; Kostarelos, K., Graphene-based electroresponsive scaffolds as polymeric implants for on-demand drug delivery. Adv. Healthc. Mater. 2014, 3 (8), 1334-1343.

[104]. Curcio, M.; Spizzirri, U. G.; Cirillo, G.; Vittorio, O.; Picci, N.; Nicoletta, F. P.; Iemma, F.; Hampel, S., On demand delivery of ionic drugs from electro-responsive cnt hybrid films. RSC Adv. 2015, 5 (56), 44902-44911.

[105]. Ramón-Azcón, J.; Ahadian, S.; Estili, M.; Liang, X.; Ostrovidov, S.; Kaji, H.; Shiku, H.; Ramalingam, M.; Nakajima, K.; Sakka, Y.; Khademhosseini, A.; Matsue, T., Dielectrophoretically aligned carbon nanotubes to control electrical and mechanical properties of hydrogels to fabricate contractile muscle myofibers. Adv. Mater. 2013, 25 (29), 4028-4034

[106]. Shin, S. R.; Jung, S. M.; Zalabany, M.; Kim, K.; Zorlutuna, P.; Kim, S. b.; Nikkhah, M.; Khabiry, M.; Azize, M.; Kong, J.; Wan, K.-t.; Palacios, T.; Dokmeci, M. R.; Bae, H.; Tang, X.; Khademhosseini, A., Carbon-nanotube-embedded hydrogel sheets for engineering cardiac constructs and bioactuators. ACS Nano 2013, 7 (3), 2369-2380.

[107]. Maggini, L.; Liu, M.; Ishida, Y.; Bonifazi, D., Anisotropically luminescent hydrogels containing magnetically-aligned MWCNTsEU(III) hybrids. Adv. Mater. 2013, 25 (17), 2462-2467.

[108]. Liu, H.-W.; Hu, S.-H.; Chen, Y.-W.; Chen, S.-Y., Characterization and drug release behavior of highly responsive chip-like electrically modulated reduced graphene oxide-poly(vinyl alcohol) membranes. J. Mat. Chem. 2012, 22 (33), 17311-17320.
[109]. Du, G.; Nie, L.; Gao, G.; Sun, Y.; Hou, R.; Zhang, H.; Chen, T.; $\mathrm{Fu}$, J., Tough and biocompatible hydrogels based on in situ interpenetrating networks of dithiol-connected graphene oxide and poly(vinyl alcohol). ACS Appl. Mater. Interfaces 2015, 7 (5), 30033008 .

[110]. Zhang, L.; Wang, Z.; Xu, C.; Li, Y.; Gao, J.; Wang, W.; Liu, Y., High strength graphene oxide/polyvinyl alcohol composite hydrogels. J. Mater. Chem. 2011, 21 (28), 10399-10406.

[111]. Sahu, A.; Choi, W. I.; Tae, G., A stimuli-sensitive injectable graphene oxide composite hydrogel. Chem. Commun. 2012, 48 (47), $5820-5822$.

[112]. Zu, S.-Z.; Han, B.-H., Aqueous dispersion of graphene sheets stabilized by pluronic copolymers: Formation of supramolecular hydrogel. J. Phys. Chem. C 2009, 113 (31), 13651-13657.

[113]. Lee, Y.; Bae, J. W.; Hoang Thi, T. T.; Park, K. M.; Park, K. D., Injectable and mechanically robust 4-arm $\mathrm{PPO}-\mathrm{PEO} /$ graphene oxide composite hydrogels for biomedical applications. Chem. Commun. 2015, 51 (42), 8876-8879.

[114]. Man, H. B.; Lam, R.; Chen, M.; Osawa, E.; Ho, D., Nanodiamondtherapeutic complexes embedded within poly(ethylene glycol) diacrylate hydrogels mediating sequential drug elution. Phys. Status Solidi A 2012, 209 (9), 1811-1818

[115]. Thompson, B. C.; Murray, E.; Wallace, G. G., Graphite oxide to graphene. Biomaterials to bionics. Adv. Mater. 2015, 27(46), 75637582

[116]. Muthoosamy, K.; G Bai, R.; Manickam, S., Graphene and graphene oxide as a docking station for modern drug delivery system. Curr. Drug Deliv. 2014, 11 (6), 701-718.

[117]. Quintana, M.; Vazquez, E.; Prato, M., Organic functionalization of graphene in dispersions. Acc. Chem. Res. 2013, 46 (1), 138-148.

[118]. Criado, A.; Melchionna, M.; Marchesan, S.; Prato, M., The covalent functionalization of graphene on substrates. Angew. Chem. Int. Ed. 2015, 54(37), 10734-10750.

[119]. Das, S.; Irin, F.; Ma, L.; Bhattacharia, S. K.; Hedden, R. C.; Green, M. J., Rheology and morphology of pristine graphene/polyacrylamide gels. ACS Appl. Mater. Interfaces 2013, 5 (17), 8633-8640

[120]. Yang, X.; Qiu, L.; Cheng, C.; Wu, Y.; Ma, Z.-F.; Li, D., Ordered gelation of chemically converted graphene for next-generation electroconductive hydrogel films. Angew. Chem. Int. Ed. 2011, 50 (32), 7325-7328

[121]. Xu, Y.; Sheng, K.; Li, C.; Shi, G., Self-assembled graphene hydrogel via a one-step hydrothermal process. ACS Nano 2010, 4 (7), 4324-4330.

[122]. Tao, C.-a.; Wang, J.; Qin, S.; Lv, Y.; Long, Y.; Zhu, H.; Jiang, Z., Fabrication of $\mathrm{pH}$-sensitive graphene oxide-drug supramolecular hydrogels as controlled release systems. J. Mat. Chem. 2012, 22 (47), 24856-24861.

[123]. Cong, H.-P.; Ren, X.-C.; Wang, P.; Yu, S.-H., Macroscopic multifunctional graphene-based hydrogels and aerogels by a metal ion induced self-assembly process. ACS Nano 2012, 6 (3), 2693-2703.

[124]. Lim, H.; Huang, N.; Lim, S.; Harrison, I.; Chia, C., Fabrication and characterization of graphene hydrogel via hydrothermal approach as a scaffold for preliminary study of cell growth. Int. J. Nanomed. 2011, 6, 1817-1823.

[125]. Lu, J.; He, Y.-S.; Cheng, C.; Wang, Y.; Qiu, L.; Li, D.; Zou, D., Self-supporting graphene hydrogel film as an experimental platform to evaluate the potential of graphene for bone regeneration. Adv. Funct. Mater. 2013, 23 (28), 3494-3502. 